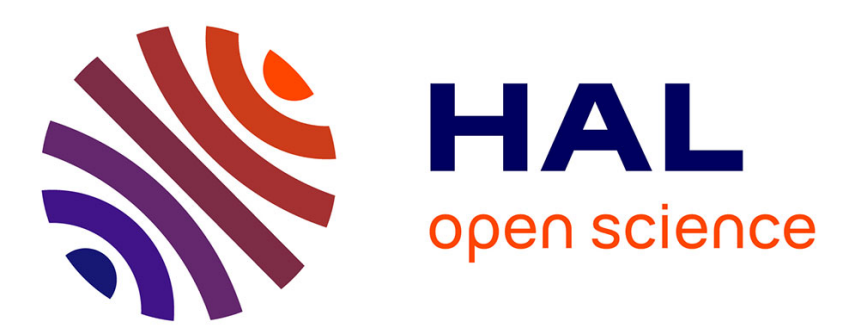

\title{
On Sensorless Induction Motor Drives: Sliding Mode Observer and Output Feedback Controller
}

\author{
Malek Ghanes, Gang Zheng
}

\section{To cite this version:}

Malek Ghanes, Gang Zheng. On Sensorless Induction Motor Drives: Sliding Mode Observer and Output Feedback Controller. IEEE Transactions on Industrial Electronics, 2009, Vol.56 ( $\left.\mathrm{N}^{\circ} 9\right)$, p. 3404-3413. inria-00531078

\section{HAL Id: inria-00531078 \\ https://hal.inria.fr/inria-00531078}

Submitted on 1 Nov 2010

HAL is a multi-disciplinary open access archive for the deposit and dissemination of scientific research documents, whether they are published or not. The documents may come from teaching and research institutions in France or abroad, or from public or private research centers.
L'archive ouverte pluridisciplinaire HAL, est destinée au dépôt et à la diffusion de documents scientifiques de niveau recherche, publiés ou non, émanant des établissements d'enseignement et de recherche français ou étrangers, des laboratoires publics ou privés. 


\title{
On Sensorless Induction Motor Drives: Sliding Mode Observer and Output Feedback Controller
}

\author{
Malek GHANES and Gang ZHENG
}

\begin{abstract}
In this paper, a sensorless output feedback controller is designed in order to drive the Induction Motor (IM) without the use of flux and speed sensors. Firstly, a new sliding mode observer that uses only the measured stator currents is synthesized to estimate the speed, the flux and the load torque. Secondly, a current-based field oriented sliding mode control is developed so as to steer the estimated speed and flux magnitude to the desired references. Stability analysis based on Lyapunov theory is also presented in order to guarantee the closed loop stability of the proposed observer-control system. Two experimental results for a $1.5-\mathrm{kW} I M$ are presented and analyzed by taking into account the unobservability phenomena of the Sensorless Induction Motor (SIM).
\end{abstract}

\section{INTRODUCTION}

Vector controlled IM drives are wide spread electromechanical conversion systems with efficient energy [4] for highdynamic performance applications, where motion control or high precision speed control is needed [22]. Since rotational transducers and their associated digital or analogue circuits give extra costs and are often complex and rather fragile, reducing the robustness of the total system, there is an increasing interest in industry in control schemes without rotational sensors-the so-called SIM control. We refer the reader to [12] for a tutorial account on the topic.

Given that high-performance controllers are readily available when speed and flux are known, it seems reasonable to estimate speed and flux, in the spirit of observer and control theories, to replace in the control scheme the actual speed and flux by its estimation. Several theoretical and practical solutions have been proposed in the literature. For instance, in [16] an algorithm for simultaneous estimation of motor speed and rotor resistance is proposed. A semi-global exponential rotor velocity and flux tracking algorithm is proved in [8]. In [17], a second-order control algorithm is designed including a rotor speed observer. An estimator of rotor speed and an indirect field-oriented control with a sliding mode are proposed in [1]. In [19], based on the model reference adaptive system (MRAS), a sensorless method estimating the rotor position and speed from the machine rotor currents is presented. Reduced and full order observers for flux and speed

Manuscript received August 26, 2008. Accepted for publication June 10, 2009.

Copyright(C) 2009 IEEE. Personal use of this material is permitted. However, permission to use this material for any other purposes must be obtained from the IEEE by sending a request to pubs-permissions@iee.org.

Malek GHANES and Gang ZHENG are with Equipe Commande des Systèmes (ECS), ENSEA, 6 Avenue du Ponceau, 95014 Cergy-Pontoise Cedex, France. Phone: 00331307366 66; Fax: 00331307366 27; e-mail:\{Ghanes, Zheng\}@ensea.fr estimation of sensorless induction-motor drives are analyzed in [11]. In [6] the problems of current decoupling control and controller tuning associated with sensorless vector-controlled induction motor drives are studied. Parallel identification schemes for both speed and stator resistance of sensorless induction motor drives are proposed in [25] for a wide range of speed estimation. Nevertheless for most of these contributions, rotor flux information and/or knowledge of load torque (and/or acknowledge load torque considered constant) are needed for controller implementation. On the other hand, removing the speed sensors affects the $I M$ observability property at very low speed. Important contributions in this direction have been reported in [9], where under some operating conditions (low speed) the $I M$ is not observable. Furthermore, strategies based on $I M$ spatial saliency methods with fundamental excitation and high frequency signal injection [12], extended Kalman filter techniques and adaptive system approaches [18] have been studied. The sensorless control of $I M$ allowing operation at very low speed can also be found in [7], [8], [9], [10], [13], [21].

The first main contribution of this paper is to design a new sliding mode observer for the SIM in presence of unknown load torque. And experimental results show the performances of the observer thanks to a dedicated Sensorless Observer Benchmark to test observers in open loop. Secondly, a sensorless control scheme is designed, which is based on a combination of field oriented control (FOC) methodology and robust sliding mode technique. Sufficient conditions are given to guarantee the stability of the whole closed-loop system. Furthermore, a Sensorless Control Benchmark is designed in order to test and to evaluate the performance of sensorless controllers. Theoretical and experimental results show the feasibility of the proposed method.

\section{OBSERVER DESIGN}

\section{A. IM Model}

In the rotating $(\mathrm{d}-\mathrm{q})$ reference frame, the $I M$ dynamic model (1) reads ([5])

$$
\left\{\begin{aligned}
\dot{\omega}_{r}= & m \phi_{r d} i_{s q}-c \omega_{r}-\frac{1}{J} T_{l} \\
\dot{\phi_{r d}}= & -a \phi_{r d}+a M_{s r} i_{s d} \\
\dot{\rho}= & p \omega_{r}+a \frac{M_{s r}}{\phi_{r d}} i_{s q} \\
\dot{i_{s d}=} & -\gamma i_{s d}+a b \phi_{r d}+p \omega_{r} i_{s q}+a \frac{M_{s r}}{\phi_{r d}} i_{s q}^{2} \\
& +m_{1} V_{s d} \\
\dot{i_{s q}}= & -\gamma i_{s q}-b p \omega_{r} \phi_{r d}-p \omega_{r} i_{s d}-a \frac{M_{s r}}{\phi_{r d}} i_{s d} i_{s q} \\
& +m_{1} V_{s q}
\end{aligned}\right.
$$


where respectively $i_{s d^{-}} i_{s q}, V_{s d^{-}} V_{s q}, \phi_{r d^{-}} \rho, \omega_{r}-T_{l}$ denote the stator currents and stator voltages, the norm of flux and angle of flux, the speed and load torque, where the subscripts $s$ and $r$ refer to the stator and rotor. The parameters $a, b, c, \gamma, \Upsilon$, $m$ and $m_{1}$ are defined by $a=\left(R_{r} / L_{r}\right), b=\left(M_{s r} / \Upsilon L_{s} L_{r}\right)$, $c=\left(f_{v} / J\right), \gamma=\left(\frac{L_{r}^{2} R_{s}+M_{s r}^{2} R_{r}}{\Upsilon L_{s} L_{r}^{2}}\right), \Upsilon=\left(1-\left(M_{s r}^{2} / L_{s} L_{r}\right)\right)$, $m=\left(p M_{s r} / J L_{r}\right), m_{1}=\left(1 / \Upsilon L_{s}\right)$, where $R_{s}$ and $R_{r}$ are the resistances, $L_{s}$ and $L_{r}$ are the self-inductances, $M_{s r}$ is the mutual inductance between the stator and rotor windings, $p$ is the number of pole-pairs, $J$ is the inertia of the system (motor and load) and $f_{v}$ is the viscous damping coefficient. The control inputs are the stator voltages. Only stator current and stator voltage are measurable. Furthermore, an operating domain $\mathcal{D}$ is defined as follows:

\section{Definition 1: Operation Domain $\mathcal{D}$ :} $\Phi_{r d}{ }^{\max }, I_{s d}^{\max }, I_{s q}^{\max }, \omega_{r}^{\max }$ and $T_{l}^{\max }$ are respectively the actual maximum values for the flux, currents, speed and load torque such that $\left|\phi_{r d}\right| \leq \Phi_{r d}^{\max },\left|i_{s d}\right| \leq$ $I_{s d}^{\max },\left|i_{s q}\right| \leq I_{s q}^{\max },\left|\omega_{r}\right| \leq \omega_{r}^{\max },\left|T_{l}\right| \leq T_{l}^{\max }$.

\section{B. Quick Review on the Observability Phenomena of SIM}

In [9], we have demonstrated that $I M$ observability cannot be established in the particular case when the fluxes $\phi_{r \alpha}, \phi_{r \beta}$ and the speed $\omega_{r}$ are constant even if the higher derivatives of outputs are used. This operating case coincides with the following physically interpretations:

1) when the fluxes are constant $\left(\dot{\phi}_{r \alpha}=\dot{\phi}_{r \beta}=0\right)$, or equivalently the excitation voltage is zero $\left(\omega_{s}=0\right)$, it implies that: $p \omega_{r}+\frac{R_{r} T_{e}}{p \phi_{d}^{2}}=\omega_{s}=0$ or $T_{e m}=-K \omega_{r}$ where $T_{e m}$ is the electromagnetic torque and $K=\frac{p^{2} \phi_{d}^{2}}{R_{r}}$;

2) if the speed motor is constant, thus $T_{e m}=\left(f_{v} \omega_{r}+T_{l}\right)^{=}=$ $-K \omega_{r}$. This last equation defines the unobservability curve in the map $\left(T_{l}, \omega_{r}\right)$ with $M=\frac{p^{2} \phi_{d}^{2}}{R_{r}}+f_{v}$ (Fig. 1) (see [9] for more details).

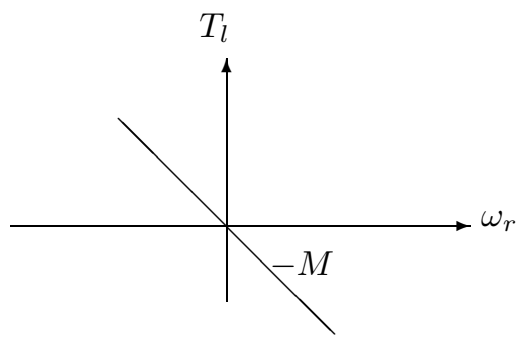

Fig. 1. Unobservability curve in the map $\left(T_{l}, \omega_{r}\right)$

Obviously, the observability is lost gradually when we approach this unobservability curve.

\section{Sliding Mode Observer design}

Several observers such as the sliding mode observer have been developed to estimate rotor speed. Sliding mode observer [2] appears as an important technique, since it offers many good properties, such as good performance against unmodeled dynamics, insensitivity to parameter variations, external disturbance rejection and fast dynamic response [23].

Since the measurements of the motor are given in the classical fixed stator frame $(a, b, c)$ while the observer is written in the frame of the rotating rotor field (d-q), it is thus necessary to carry out a change of reference from the measures. Initial measurements are transformed from the three-phase reference frame to a diphasic reference frame by using the following Concordia equations:

$$
\left\{\begin{aligned}
V_{\alpha} & =\sqrt{\frac{2}{3}}\left(V_{a}-\frac{1}{2} V_{b}-\frac{1}{2} V_{c}\right) ; & V_{\beta} & =\sqrt{\frac{2}{3}}\left(V_{b}-V_{c}\right) \\
i_{\alpha} & =\sqrt{\frac{2}{3}}\left(i_{a}-\frac{1}{2} i_{b}-\frac{1}{2} i_{c}\right) ; & i_{\beta} & =\sqrt{\frac{2}{3}}\left(i_{b}-i_{c}\right)
\end{aligned}\right.
$$

where $V_{a}, V_{b}, V_{c}$ and $i_{a}, i_{b}, i_{c}$ are the three-phase voltages of $I M$ and three-phase currents (supposed balanced).

The next step consists in passing in the turning reference frame by the Park transformation. This transformation requires the calculation of rotor field frame angle with respect to the fixed reference frame. This calculation is carried out starting from the equations of (2) to (4), just as the calculation of the new measurements to the frame (d-q).

$$
\begin{aligned}
\frac{d \hat{\rho}}{d t} & =p \hat{\omega}_{r}+\alpha_{r} \frac{M_{s r}}{\hat{\phi}_{r d}} i_{s q} \\
V_{s d} & =\cos (\hat{\rho}) V_{\alpha}+\sin (\hat{\rho}) V_{\beta} \\
V_{s q} & =-\cos (\hat{\rho}) V_{\alpha}+\sin (\hat{\rho}) V_{\beta}
\end{aligned}
$$

Let us consider system (1) with the outputs $y=\left(\begin{array}{c}i_{s d} \\ i_{s q}\end{array}\right)$. It is easy to check that $\phi_{r d}$ and $\omega_{r}$ of (1) is not observable. Hence classical observer design techniques cannot be applied. However, we notice that (1) has a stable zero dynamics [14] about $\phi_{r d}$, with the output $y_{1}=i_{s d}$ and $a>0$ in (1), hence by simply using an estimator, it is possible to estimate or detect $\phi_{r d}$. With the estimation of $\phi_{r d}$, then $\omega_{r}$ of (1) becomes observable. Hence we can use a sliding mode observer to recover $\omega_{r}$.

Remark 1: Since system (1) stays in $\mathcal{D}$ defined in Definition 1, thus it is Bounded Input Bounded State (BIBS).

For system (1), we propose the following sliding mode observer:

$$
\left\{\begin{aligned}
\dot{\hat{\phi}}_{r d}= & -a \hat{\phi}_{r d}+a M_{s r} i_{s d} \\
\dot{\hat{\imath}}_{s d}= & -\gamma i_{s d}+E_{1} a b \hat{\phi}_{r d}+E_{1} a \frac{M_{s r}}{\hat{\phi}_{r d}} i_{s q}^{2} \\
& +m_{1} V_{s d}+E_{1} \lambda_{1} s g n\left(i_{s d}-\hat{\imath}_{s d}\right) \\
\dot{\hat{\omega}}_{r}= & E_{1} m \hat{\phi}_{r d} i_{s q}-E_{2} c \tilde{\omega}_{r}+E_{2} \lambda_{2} \operatorname{sgn}\left(\tilde{\omega}_{r}-\hat{\omega}_{r}\right)
\end{aligned}\right.
$$

where

$$
\operatorname{sgn}(*): \begin{cases}1 & \text { if } *>0 \\ -1 & \text { if } *<0 \\ \in[-1,1] & \text { if } *=0\end{cases}
$$

with the auxiliary state $\tilde{\omega}_{r}=\frac{E_{2} \lambda_{1} \operatorname{sgn}\left(i_{s d}-\hat{\imath}_{s d}\right)}{p i_{s q}}$ where

$$
\begin{gathered}
E_{1}= \begin{cases}0, & \text { if }\left|\hat{\phi}_{r d}-\phi_{r d}\right|>\epsilon \\
1, & \text { if }\left|\hat{\phi}_{r d}-\phi_{r d}\right| \leq \epsilon ;\end{cases} \\
E_{2}= \begin{cases}0, & \text { if } \hat{\imath}_{s d} \neq i_{s d} \\
1, & \text { if } \hat{\imath}_{s d}=i_{s d} ;\end{cases}
\end{gathered}
$$

where $\epsilon$ is a small positive real and the estimation of load torque $T_{l}$ given by the following equation

$$
\tilde{T}_{l}=-E_{3} J \lambda_{2} \operatorname{sgn}\left(\tilde{\omega}_{r}-\hat{\omega}_{r}\right)
$$


where $E_{3}= \begin{cases}0, & \text { if } \hat{\omega}_{r} \neq \tilde{\omega}_{r} \\ 1, & \text { if } \hat{\omega}_{r}=\tilde{\omega}_{r}\end{cases}$

Theorem 1: For system (1), the proposed observer (5) can exponentially converge to $\phi_{r d}$ and estimate $\omega_{r}$ and $T_{l}$.

Proof: Assuming that $E_{1}=0$ (if $E_{1}=1$ we directly move to the next step), the observation error dynamics of $\phi_{r d}$, noted as $\varepsilon_{\phi_{r d}}=\phi_{r d}-\hat{\phi}_{r d}$, is defined as: $\dot{\varepsilon}_{\phi_{r d}}=-a \varepsilon_{\phi_{r d}}$ with $a>0$, and it implies exponential convergence of $\hat{\phi}_{r d}$ to $\phi_{r d}$.

Given a sufficient small real $\epsilon>0$, we can always find $t_{1}$, such that when $t>t_{1}$, we have $\varepsilon_{\phi_{r d}} \leq \epsilon$, hence we have $E_{1}=1$ after $t>t_{1}$. Then we consider the convergence of $\hat{\imath}_{s d}$ to $i_{s d}$. For this, we note $\varepsilon_{i_{s d}}=i_{s d}-\hat{\imath}_{s d}$. Since when $t>t_{1}$ we have $E_{1}=1$ and $\varepsilon_{\phi_{r d}} \leq \epsilon$ where $\epsilon$ is a sufficient small real, then

$$
\dot{\varepsilon}_{i_{s d}}=\left(a b-\frac{a M_{s r} i_{s q}^{2}}{\phi_{r d} \hat{\phi}_{r d}}\right) \varepsilon_{\phi_{r d}}+p \omega_{r} i_{s q}-\lambda_{1} \operatorname{sgn}\left(i_{s d}-\hat{\imath}_{s d}\right)
$$

Let us consider the following Lyapunov function: $V_{\varepsilon_{i_{s d}}}=$ $\frac{1}{2} \varepsilon_{i_{s d}}^{2}$. Since system (1) stays in $\mathcal{D}$, hence if

$$
\lambda_{1}=\max \left\{p \omega_{r} i_{s q}+\left(a b-\frac{a M_{s r} i_{s q}^{2}}{\phi_{r d} \hat{\phi}_{r d}}\right) \varepsilon_{\phi_{r d}}\right\}+\zeta_{i_{s d}}
$$

with $\zeta_{i_{s d}}>0$ and $\varepsilon_{\phi_{r d}} \leq \epsilon$, we have

$$
\begin{aligned}
\dot{V}_{\varepsilon_{i_{s d}}} & =\left(p \omega_{r} i_{s q}+\left(a b-\frac{a M_{s r} i_{s_{q}}^{2}}{\phi_{r d} \hat{\phi}_{r d}}\right) \varepsilon_{\phi_{r d}}-\lambda_{1} \operatorname{sgn}\left(\varepsilon_{i_{s d}}\right)\right) \varepsilon_{i_{s d}} \\
& \leq-\zeta_{i_{s d}}\left|\varepsilon_{i_{s d}}\right|=-\sqrt{2} \zeta_{i_{s d}} V_{\varepsilon_{\phi_{r d}}}^{1 / 2}
\end{aligned}
$$

which implies the convergence of $\hat{\imath}_{s d}$ to $i_{s d}$ in a finite time, noted as $t_{2}$. Hence after $t>t_{2}>t_{1}$, we have $E_{2}=1$ and $\dot{\varepsilon}_{i_{s d}}=\varepsilon_{i_{s d}}=0$, which gives $p \omega_{r} i_{s q}+$ $\left(a b-\frac{a M_{s r} i_{s q}^{2}}{\phi_{r d} \hat{\phi}_{r d}}\right) \varepsilon_{\phi_{r d}}-\lambda_{1} \operatorname{sgn}\left(i_{s d}-\hat{\imath}_{s d}\right)=0$. Finally we have $\tilde{\omega}_{r}=\omega_{r}+\left(a b-\frac{a M_{s r} i_{s q}^{2}}{\phi_{r d} \hat{\phi}_{r d}}\right) \frac{\varepsilon_{\phi_{r d}}}{p i_{s q}}=\omega_{r}+\mathcal{E}(t)$ where $\mathcal{E}(t)=\left(a b-\frac{a M_{s r} i_{s q}^{2}}{\phi_{r d} \hat{\phi}_{r d}}\right) \frac{\varepsilon_{\phi_{r d}}}{p i_{s q}}$. Since system (1) stays in $\mathcal{D}$ defined in Definition 1, it can be seen that $\mathcal{E}(t)$ and $\dot{\mathcal{E}}(t)$ are bounded and they converge to zero exponentially. Hence we have the exponential convergence of $\tilde{\omega}_{r}$ to $\omega_{r}$.

Consequently we have

$$
\dot{\tilde{\omega}}_{r}=\dot{\omega}_{r}+\dot{\mathcal{E}}(t)=m \phi_{r d} i_{s q}-c \omega_{r}-\frac{1}{J} T_{l}+\dot{\mathcal{E}}(t)
$$

Analogously, by taking the Lyapunov function: $V_{\varepsilon_{\omega_{r}}}=\frac{1}{2} \varepsilon_{\omega_{r}}^{2}$ with $\varepsilon_{\omega_{r}}=\tilde{\omega}_{r}-\hat{\omega}_{r}$, and if

$$
\lambda_{2}=\max \left\{-\frac{1}{J} T_{l}+m i_{s q} \varepsilon_{\phi_{r d}}+c \mathcal{E}(t)+\dot{\mathcal{E}}(t)\right\}+\zeta_{\omega_{r}}
$$

with $\zeta_{\omega_{r}}>0$, we have

$$
\begin{aligned}
\dot{V}_{\varepsilon_{\omega_{r}}}= & \left(-\frac{1}{J} T_{l}+m i_{s q} \varepsilon_{\phi_{r d}}+c \mathcal{E}(t)+\dot{\mathcal{E}}(t)\right) \varepsilon_{\omega_{r}} \\
& -\lambda_{2} \operatorname{sgn}\left(\tilde{\omega}_{r}-\hat{\omega}_{r}\right) \varepsilon_{\omega_{r}} \\
\leq & -\zeta_{\omega_{r}}\left|\varepsilon_{\omega_{r}}\right|=-\sqrt{2} \zeta_{\omega_{r}} V_{\varepsilon_{\omega_{r}}}^{1 / 2}
\end{aligned}
$$

which signifies the convergence of $\hat{\omega}_{r}$ to $\tilde{\omega}_{r}$ in a finite time, noted as $t_{3}$. Hence after $t>t_{3}>t_{2}$, we have $E_{3}=1$ and $\dot{\varepsilon}_{\omega_{r}}=\varepsilon_{\omega_{r}}=0$, which gives $\tilde{T}_{l}=T_{l}-J m i_{s q} \varepsilon_{\phi_{r d}}-J c \mathcal{E}(t)-$ $J \dot{\mathcal{E}}(t)$. Since $\tilde{\omega}_{r}$ and $\hat{\phi}_{r d}$ converge exponentially to $\omega_{r}$ and $\phi_{r d}$ respectively, which implies $\varepsilon_{\phi_{r d}}, \mathcal{E}(t)$ and $\dot{\mathcal{E}}(t)$ vanish exponentially. Consequently $\tilde{T}_{l}$ converges exponentially to $T_{l}$.

\section{FOC via Sliding Mode Techniques}

In this section, a controller is designed by combining the FOC method ([3]) with Sliding Mode Control method (SMC, [23], [20]). The design procedure is based on the well-known assumption of current-fed IM (see [15],[5]).

A. Field Oriented Control. Consider the IM dynamic model given by (1) in the (d-q) reference frame. In this frame the electromagnetic torque $T_{e m}=\frac{p M_{s r}}{L_{r}} \phi_{r d} i_{s q}$ is proportional to the product of $\phi_{r d}$ and $i_{s q}$. Thus by holding constant the magnitude of the rotor flux, a linear relation between $i_{s q}$ and $T_{e m}$ is obtained. In order to cancel the nonlinear dynamics of $i_{s d}$ and $i_{s q}$, the system is forced into current-command mode by using high gain feedback (see [15],[5]). More precisely, the following PI current controllers

$$
\left\{\begin{array}{l}
V_{s d}=K i_{v d} \int_{0}^{t}\left(i_{s d}^{*}-i_{s d}\right) d t+K p_{v d}\left(i_{s d}^{*}-i_{s d}\right) \\
V_{s q}=K i_{v q} \int_{0}^{t}\left(i_{s q}^{*}-i_{s q}\right) d t+K p_{v q}\left(i_{s q}^{*}-i_{s q}\right)
\end{array}\right.
$$

are used to force $i_{s d}$ and $i_{s q}$ to track their respective references $i_{s d}^{*}$ and $i_{s q}^{*}$ and produce fast responses when large feedback gains are used. Hence, assuming that $i_{s d}^{*}$ and $i_{s q}^{*}$ as the new inputs, it follows that

$$
\left\{\begin{array}{l}
\dot{\omega}_{r}=m \phi_{r d} i_{s q}^{*}-c \omega_{r}-\frac{T_{l}}{J} \\
\dot{\phi}_{r d}=-a \phi_{r d}+a M_{s r} i_{s d}^{*}
\end{array}\right.
$$

In order to solve the flux and speed trajectory tracking problem, the following assumption is introduced.

Assumption 1: a- The state initial conditions of the $I M$ are in the physical domain $\mathcal{D}$.

b- The desired trajectories $\left(\phi_{r d}^{*}\right.$ and $\left.\omega_{r}^{*}\right)$ are in the physical domain $\mathcal{D}$.

c- The actual load torque is assumed to be bounded by a maximal fixed value $\varrho$. This maximal value is chosen in accordance to the realistic torque characteristics of the chosen drive: $\left|T_{l}\right|<\varrho$.

\section{B. Sliding Mode Control.}

Flux controller design. From (11), consider the following $I M$ flux dynamic equation with uncertainties

$$
\dot{\phi}_{r d}=-a \phi_{r d}+\Delta a \phi_{r d}+\kappa i_{s d}^{*}
$$

where $\kappa=a M_{s r}$ and $\Delta a$ is the uncertainty term of parameter $a$. In order to design a flux sliding mode controller, we define the flux tracking error $e_{\phi_{r d}}=\phi_{r d}-\phi_{r d}^{*}$ where $\phi_{r d}^{*}$ is the flux reference. Then the associated error dynamics is

$$
\dot{e}_{\phi_{r d}}=-a e_{\phi_{r d}}+\kappa i_{s d}^{*}-a \phi_{r d}^{*}-\dot{\phi}_{r d}^{*}+\Delta a \phi_{r d}
$$

¿From $S M C$ theory, let us define the $\phi_{r d}$ flux sliding manifold as follows

$$
\sigma_{\phi_{r d}}=e_{\phi_{r d}}-\left(k_{\phi_{r d}}-a\right) \int_{0}^{t} e_{\phi_{r d}}(\tau) d \tau .
$$

Then the associated Lyapunov function is selected as $V_{\sigma_{\phi_{r d}}}=$ $\frac{1}{2} \sigma_{\phi_{r d}}^{2}$, where its time derivative is given by

$$
\dot{V}_{\sigma_{\phi_{r d}}}=\sigma_{\phi_{r d}}\left[\sigma_{\phi_{r d} 1}+\sigma_{\phi_{r d} 2} i_{s d}^{*}+\Delta a \phi_{r d}\right]
$$


with $\sigma_{\phi_{r d} 1}=-k_{\phi_{r d}} e_{\phi_{r d}}-a \phi_{r d}^{*}-\dot{\phi}_{r d}^{*}$ and $\sigma_{\phi_{r d} 2}=\kappa$. Therefore, the sliding mode controller can be designed as follows

$$
i_{s d, e q}^{*}=-\frac{l_{\phi_{r d}} \sigma_{\phi_{r d}}+\sigma_{\phi_{r d} 1}+\eta_{\phi_{r d}} \operatorname{sgn}\left(\sigma_{\phi_{r d}}\right)}{\sigma_{\phi_{r d}} 2}
$$

with the sign function is defined by (6).

Consequently, (14) becomes

$$
\dot{V}_{\sigma_{\phi_{r d}}}=-l_{\phi_{r d}} V_{\sigma_{\phi_{r d}}}+\sigma_{\phi_{r d}}\left[-\eta_{\phi_{r d}} \operatorname{sgn}\left(\sigma_{\phi_{r d}}\right)+\Delta a \phi_{r d}\right] \text {. }
$$

Choosing $l_{\phi_{r d}}>0$ and $\eta_{\phi_{r d}}>\max \left\{\left\|\Delta a \phi_{r d}\right\|\right\}$ (defined hereafter), it follows that $\dot{V}_{\sigma_{\phi_{r d}}} \leq 0$. As $V_{\sigma_{\phi_{r d}}}$ is contracting. ¿From Assumption (1-a-b), then $\max \left\{\phi_{r d}\right\}:=K_{\phi_{r d}}^{\max }$ can not be greater than $\max \left\{\phi_{r d}(0), \phi_{r d}^{*}\right\}+\left|\Delta e_{\phi_{r d}}(0)\right|$. Consequently, $\Delta a \phi_{r d}$ is bounded and can be set as

$$
\eta_{\phi_{r d}}=\Delta a^{\max } K_{\phi_{r d}}^{\max }+b_{\phi_{r d}}
$$

with $b_{\phi_{r d}}$ a small positive constant. Hence (16) becomes

$$
\begin{aligned}
\dot{V}_{\sigma_{\phi_{r d}}} & =-l_{\phi_{r d}} V_{\sigma_{\phi_{r d}}}-b_{\phi_{r d}}\left|\sigma_{\phi_{r d}}\right| \\
& <-\sqrt{2} b_{\phi_{r d}} V^{1 / 2}
\end{aligned}
$$

which implies that we have $\sigma_{\phi_{r d}}=0$ in a finite time. Therefore (13) becomes

$$
\dot{e}_{\phi_{r d}}=\left(k_{\phi_{r d}}-a\right) e_{\phi_{r d}} .
$$

Hence, the flux tracking error $e_{\omega_{r}}$ exponentially converges to 0 for $\left(k_{\phi_{r d}}-a\right)<0$.

Choosing (15) to force $\phi_{r d}$ to track its reference $\phi_{r d}^{*}$ ensures that the flux is properly established in the motor. Hence, after the $I M$ is fluxed $\left(\phi_{r d}=\phi_{r d}^{*}=\right.$ constant $)$, the electromagnetic torque $\left(T_{e m}\right)$ can be rewritten as $T_{e m}=K_{T} i_{s q}^{*}$, where $K_{T}$ is the motor torque constant defined by $K_{T}=\frac{p M_{s r}}{L_{r}} \phi_{r d}$. As a consequence, the linear relationship between the input $i_{s q}^{*}$ and the speed dynamics $\dot{\omega}_{r}$ is obtained. Then, the speed control is obtained through the input $i_{s q}^{*}$ via a speed controller described below.

Speed controller design. Consider the mechanical equation of (11) including uncertainties as follows

$$
\dot{\omega}_{r}=-c \omega_{r}+h i_{s q}^{*}+d_{\omega_{r}}
$$

where $h=m \phi_{r d}$ and $d_{\omega_{r}}=-\Delta c \omega_{r}-\frac{T_{l}}{J}$ is the term uncertainty. Defining the speed tracking error $e_{\omega_{r}}=\omega_{r}-\omega_{r}^{*}$, it follows

$$
\dot{e}_{\omega_{r}}=-c e_{\omega_{r}}+h i_{s q}^{*}-c \omega_{r}^{*}-\dot{\omega}_{r}^{*}+d_{\omega_{r}}
$$

Define now the sliding manifold as follows

$$
\sigma_{\omega_{r}}=e_{\omega_{r}}-\left(k_{\omega_{r}}-c\right) \int_{0}^{t} e_{\omega_{r}}(\tau) d \tau
$$

and the candidate Lyapunov function associated to the sliding manifold (21) is defined as $V_{\sigma_{\omega_{r}}}=\frac{1}{2} \sigma_{\omega_{r}}^{2}$. By computing its time derivative, we obtain

$$
\dot{V}_{\sigma_{\omega_{r}}}=\sigma_{\omega_{r}} \sigma_{\omega_{r}}=\sigma_{\omega_{r}}\left[\sigma_{\omega_{r} 1}+\sigma_{\omega_{r} 2} i_{s q}^{*}+d_{\omega r}\right]
$$

where $\sigma_{\omega_{r} 1}=-k e_{\omega_{r}}-c \omega_{r}^{*}-\dot{\omega}_{r}^{*}$ and $\sigma_{\omega_{r} 2}=h$.
Then, the speed controller reads

$$
i_{s q, e q}^{*}=-\frac{l_{\omega_{r}} \sigma_{\omega_{r}}+\sigma_{\omega_{r} 1}+\eta_{\omega_{r}} \operatorname{sgn}\left(\sigma_{\omega_{r}}\right)}{\sigma_{\omega_{r} 2}}
$$

with the sign function is defined by (6). Then (22) becomes

$$
\dot{V}_{\sigma_{\omega_{r}}}=-l_{\omega_{r}} V_{\sigma_{\omega_{r}}}+\sigma_{\omega_{r}}\left[-\eta_{\omega_{r}} \operatorname{sgn}\left(\sigma_{\omega_{r}}\right)+d_{\omega_{r}}\right]
$$

By choosing $l_{\omega_{r}}>0$ and $\eta_{\omega_{r}}>\max \left\{\left\|d_{\omega_{r}}\right\|\right\}$ (defined hereafter). From Assumption (1-a-b) then $\max \left\{\omega_{r}\right\}:=K_{\omega_{r}}^{\max }$ can not be greater than $\max \left\{\left|\omega_{r}(0)\right|,\left|\omega_{r}^{*}\right|\right\}+\left|\Delta e_{\omega_{r}}(0)\right|$. Consequently, as $d_{\omega_{r}}=\Delta c \omega_{r}+\frac{T_{l}}{J}$ then $d_{\omega_{r}}$ is bounded. Finally, from Assumption (1-c) $\eta_{\omega_{r}}$ is set as

$$
\eta_{\omega_{r}}=\Delta c K_{\omega_{r}}^{\max }+\frac{\varrho}{J}+b_{\omega_{r}}
$$

with $b_{\omega_{r}}$ a small positive constant. Following the same procedure used for flux, we have $\dot{V}_{\sigma_{\omega_{r}}}<-\sqrt{2} b_{\omega_{r}} V^{1 / 2}$, which means we obtain $\sigma_{\omega_{r}}=0$ in a finite time. Therefore (20) implies

$$
\dot{e}_{\omega_{r}}=\left(k_{\omega_{r}}-c\right) e_{\omega_{r}}
$$

which makes that the speed tracking error $e_{\omega_{r}}$ exponentially converges to 0 for $\left(k_{\omega_{r}}-c\right)<0$.

Proposition 1: Consider IM model (11) and suppose that Assumption 1 is satisfied. Then under the action of speed controller (22) and flux controller (15), the rotor speed and the flux track their desired trajectories exponentially.

Proof: Using $V_{c}=\frac{1}{2} \sigma_{\phi_{r d}}^{2}+\frac{1}{2} \sigma_{\omega_{r}}^{2}$ as a Lyapunov function candidate, then the time derivative gives

$$
\begin{aligned}
\dot{V}_{c}= & -l_{\phi_{r d}} V_{\sigma_{\phi_{r d}}}+\left[-\eta_{\phi_{r d}} \operatorname{sgn}\left(\sigma_{\phi_{r d}}\right)+\Delta a \phi_{r d}\right] \\
& -l_{\omega_{r}} V_{\sigma_{\omega_{r}}}+\left[-\eta_{\omega_{r}} \operatorname{sgn}\left(\sigma_{\omega_{r}}\right)+d_{\omega_{r}}\right] \\
< & -\sqrt{2} b V^{1 / 2}
\end{aligned}
$$

where $b=\min \left\{b_{\phi_{r d}}, b_{\omega_{r}}\right\}$. It implies that after a finite time we have $\dot{e}_{\phi_{r d}}=\left(k_{\phi_{r d}}-a\right) e_{\phi_{r d}}$ and $\dot{e}_{\omega_{r}}=\left(k_{\omega_{r}}-c\right) e_{\omega_{r}}$, hence we have exponential convergence of the rotor speed and flux.

\section{STABILITY ANALYSIS OF THE CLOSED-LOOP SYSTEM}

In order to implement controllers (15) and (22), the speed/flux measures are replaced by their estimates resulting in the new controllers

$i_{s d}^{*}\left(\hat{\phi}_{r d}\right)=\frac{-l_{\phi r d} \sigma_{\hat{\phi}_{r d}}+k_{\phi r d} e_{\hat{\phi}_{r d}}+a \phi_{r d}^{*}+\dot{\phi}_{r d}^{*}-\eta_{\phi r d} \operatorname{sgn}\left(\sigma_{\hat{\phi}_{r d}}\right)}{\kappa}$

and

$$
i_{s q}^{*}\left(\hat{\omega}_{r}\right)=\frac{-l_{\omega r} \sigma_{\hat{\omega}_{r}}+k_{\omega_{r}} e_{\hat{\omega}_{r}}+c \omega_{r}^{*}+\dot{\omega}_{r}^{*}-\eta_{\omega r} \operatorname{sgn}\left(\sigma_{\hat{\omega}_{r}}\right)}{m \hat{\phi}_{r d}}
$$

where

$$
\left\{\begin{aligned}
e_{\hat{\phi}_{r d}} & =\hat{\phi}_{r d}-\phi_{r d}^{*} \\
e_{\hat{\omega}_{r}} & =\hat{\omega}_{r}-\omega_{r}^{*} \\
\sigma_{\hat{\phi}_{r d}} & =e_{\hat{\phi}_{r d}}-\left(k_{\phi_{r d}}-a\right) \int_{0}^{t} e_{\hat{\phi}_{r d}} d \tau \\
\sigma_{\hat{\omega}_{r}} & =e_{\hat{\omega}_{r}}-\left(k_{\omega_{r}}-c\right) \int_{0}^{t} e_{\hat{\omega}_{r}} d \tau
\end{aligned}\right.
$$

with the speed $\hat{\omega}_{r}$ and the flux $\hat{\phi}_{r d}$ are given by observer (5).

The $I M$ observer must be fluxed to ensure estimated speed tracking. In order to avoid the singularity in (26), the flux (5) is initialized with initial conditions different from zero. In practice, electrical engineers overcome this singularity by 
starting to track firstly the flux $\phi_{r d}$ to its reference $\phi_{r d}^{*}=$ constant. The same trick is adopted for the estimated flux $\hat{\phi}_{r d}$ by adding an offset $\varepsilon=0.05 \mathrm{~Wb}$ such as

$$
i_{s q}^{*}\left(\hat{\omega}_{r}\right)=\frac{-l_{\omega r} \sigma_{\hat{\omega}_{r}}+k_{\omega_{r}} e_{\hat{\omega}_{r}}+c \omega_{r}^{*}+\dot{\omega}_{r}^{*}-\eta_{\omega r} \operatorname{sgn}\left(\sigma_{\hat{\omega}_{r}}\right)}{\max \left\{\hat{\phi}_{r d}, \varepsilon\right\} m}
$$

Theorem 2: Consider system (1) where (5) is an associated sliding mode observer which is initialized in $\mathcal{D}$, suppose that Assumption (1-a-b) holds. Under the action of controllers defined in (25) and (27), the tracking errors of speed and flux converge asymptotically to zero.

Proof: By choosing the whole Lyapunov function as $V=V_{1}+V_{2}$ where $V_{1}=\frac{1}{2} \sigma_{\hat{\phi}_{r d}}^{2}+\frac{1}{2} \varepsilon_{\phi_{r d}}^{2}$ and $V_{2}=\frac{1}{2} \sigma_{\hat{\omega}_{r}}^{2}+$ $\frac{1}{2} \varepsilon_{\omega_{r}}^{2}$, then we have $\dot{V}=\dot{V}_{1}+\dot{V}_{2}$ where

$$
\begin{aligned}
\dot{V}_{1}= & \sigma_{\hat{\phi}_{r d}} \dot{\sigma}_{\hat{\phi}_{r d}}+\varepsilon_{\phi_{r d}} \dot{\varepsilon}_{\phi_{r d}} \\
= & \sigma_{\phi_{r d}}\left(\dot{e}_{\hat{\phi}_{r d}}-\left(k_{\phi_{r d}}-a\right) e_{\hat{\phi}_{r d}}\right)+\varepsilon_{\phi_{r d}}\left(\dot{\phi}_{r d}-\dot{\hat{\phi}}_{r d}\right) \\
\leq & \sigma_{\hat{\phi}_{r d}}\left(-a e_{\hat{\phi}_{r d}}+\kappa i_{s d}^{*}\left(\hat{\phi}_{r d}\right)-a \phi_{r d}^{*}-\dot{\phi}_{r d}^{*}+\Delta a \hat{\phi}_{r d}\right) \\
& -\left(k_{\phi_{r d}}-a\right) \sigma_{\hat{\phi}_{r d}} e_{\hat{\phi}_{r d}}-a \varepsilon_{\phi_{r d}}^{2} \\
= & \sigma_{\hat{\phi}_{r d}}\left(-l_{\phi r d} \sigma_{\hat{\phi}_{r d}}+\Delta a \phi_{r d}-\eta_{\phi r d} \operatorname{sgn}\left(\sigma_{\hat{\phi}_{r d}}\right)\right)-a \varepsilon_{\phi_{r d}}^{2} \\
\leq & -l_{\phi r d} \sigma_{\hat{\phi}_{r d}}^{2}-b_{\phi_{r d}}\left|\sigma_{\hat{\phi}_{r d}}\right|-a \varepsilon_{\phi_{r d}}^{2}<-\mu_{1} V_{1}
\end{aligned}
$$

with $\mu_{1}=\min \left\{2 l_{\phi r d}, 2 a\right\}$ and

$$
\begin{aligned}
\dot{V}_{2}= & \sigma_{\hat{\omega}_{r}} \dot{\sigma}_{\hat{\omega}_{r}}+\dot{\varepsilon}_{\omega_{r}} \varepsilon_{\omega_{r}} \\
= & \sigma_{\hat{\omega}_{r}}\left(\dot{e}_{\hat{\omega}_{r}}-\left(k_{\omega_{r}}-c\right) e_{\hat{\omega}_{r}}\right)+\varepsilon_{\omega_{r}}\left(\dot{\omega}_{r}-\dot{\hat{\omega}}_{r}\right) \\
= & \sigma_{\hat{\omega}_{r}}\left(-c e_{\hat{\omega}_{r}}+h i_{s q}^{*}\left(\hat{\omega}_{r}\right)-c \omega_{r}^{*}-\dot{\omega}_{r}^{*}+d_{\hat{\omega}_{r}}\right) \\
& -\left(k_{\omega_{r}}-c\right) \sigma_{\hat{\omega}_{r}} e_{\hat{\omega}_{r}}-\zeta_{\omega_{r}}\left|\varepsilon_{\omega_{r}}\right| \\
= & \sigma_{\hat{\omega}_{r}}\left[-l_{\omega_{r}} \sigma_{\hat{\omega}_{r}}+d_{\hat{\omega}_{r}}-\eta_{\omega_{r}} \operatorname{sgn}\left(\sigma_{\hat{\omega}_{r}}\right)\right]-\zeta_{\omega_{r}}\left|\varepsilon_{\omega_{r}}\right| \\
\leq & -l_{\omega_{r}} \sigma_{\hat{\omega}_{r}}^{2}-b_{\omega_{r}}\left|\sigma_{\hat{\omega}_{r}}\right|-\zeta_{\omega_{r}}\left|\varepsilon_{\omega_{r}}\right|<-\mu_{2} V_{2}^{1 / 2}
\end{aligned}
$$

with $\mu_{2}=\min \left\{\sqrt{2} b_{\omega_{r}}, \sqrt{2} \zeta_{\omega_{r}}\right\}$ since $\sqrt{|x|}+\sqrt{|y|} \geq$ $\sqrt{|x|+|y|}$. Hence we have

$$
\dot{V}<-\mu_{1} V_{1}-\mu_{2} V_{2}^{1 / 2} \leq 0
$$

which implies asymptotical convergence of speed and flux to zero.

\section{EXPERIMENTAL RESULTS}

Here, the tests have been performed with the following $I M$ values:

\begin{tabular}{|c|c||c|c|}
\hline Nominal rate power & $1.5 \mathrm{~kW}$ & $R_{s}$ & $1.47 \Omega$ \\
\hline Nominal speed & $1430 \mathrm{rpm}$ & $R_{r}$ & $0.79 \Omega$ \\
\hline Number of pole pairs & 2 & $L_{s}$ & $0.105 \mathrm{H}$ \\
\hline Nominal voltage & $220 \mathrm{~V}$ & $L_{r}$ & $0.094 \mathrm{H}$ \\
\hline Nominal current & $6.1 \mathrm{~A}$ & $\mathrm{~J}$ & $0.0077 \mathrm{Kg} . \mathrm{m}^{2}$ \\
\hline Sampling time & $200 \mu \mathrm{s}$ & $f_{v}$ & $0.0029 \frac{\mathrm{Nm} . \mathrm{s}}{\mathrm{rad}}$ \\
\hline
\end{tabular}

\section{A. Part I: Observer experimental results}

Before evaluating the performances of the proposed observer-controller in closed loop, it is necessary to test and validate the performances of the proposed observer in open loop. For that a dedicated benchmark (Fig. 7) is defined to test observers on and near the unobservability curve ${ }^{1}$.

\footnotetext{
${ }^{1}$ see section II-B for more details
}

Zone 1. The initial conditions of speed and stator pulsation are such that the IM is observable (from 0 s to $2 \mathrm{~s}$ ).

Zone 2. The stator voltages is forced to zero corresponding to constant fluxes while the rotor velocity remains constant, making the state unobservable between 4 and 5 seconds and between 6 and 7 seconds.

Zone 3. Between 5 and 6 seconds, the rotor moves with a constant acceleration, allowing to check the observer convergence when the state is slightly observable.

Zone 4. The IM is driven outside the unobservability conditions. Practically, to apply this benchmark, the main difficulty lies in the simultaneous control of speed and stator pulsation so that the slip pulsation $\omega_{g}=\omega_{s}-p \omega$ does not exceed a limiting value $\omega_{g}=R_{r} M_{s r} i_{q} / L_{r} \phi_{d}$, which corresponds to the highest admissible stator current. The reference slip pulsation is given in Fig.3.c. In order to respect the above condition, it is necessary to drive the speed of the motor by a connected synchronous motor which is controlled to follow the speed trajectory. Simultaneously, the frequency of the voltages applied to the $I M$ stator follows the stator pulsation reference shown in Fig.3. Moreover robustness tests are defined by realistic variation of stator resistance and stator inductance. This benchmark is applied on an experimental setup. The frequency of the voltages applied to the stator of $I M$ is controlled by classical U/f control which is independent of motor measurements and estimated state. At the same time, the speed of the $I M$ is controlled by the connected synchronous motor using speed measurement. The sliding mode observer uses only the measurement of stator voltages and stator currents.

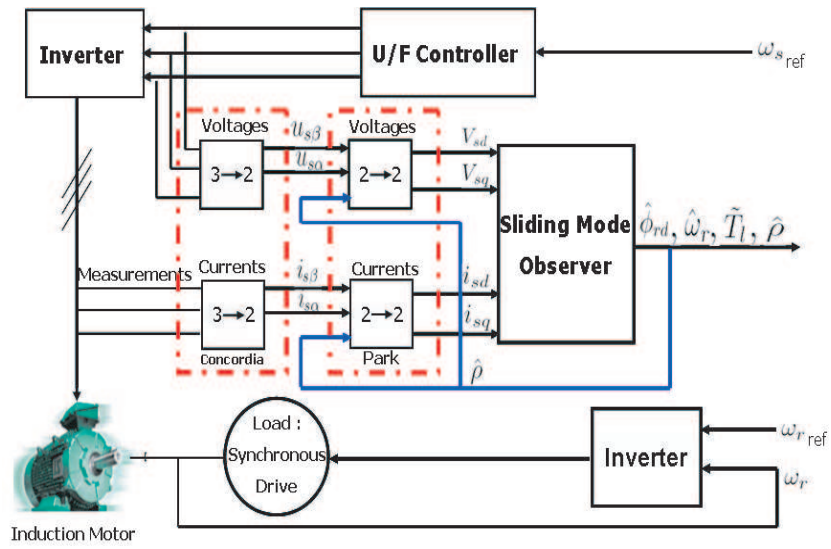

Fig. 2. Pie chart of the sliding mode observer.

The block scheme used in experimental set-up to test the sliding mode observer in open loop is presented in Fig. 2. The block 'sliding mode observer' uses only the stator current measurements in the reference rotating frame $(d-q)$ to estimate the speed, flux, load torque and the angle of flux (after using the transformation of Concordia and Park). The block 'U/f Controller' provides classical U/f control which is independent of motor measurements and estimated state. This block controls the frequency of the voltages applied to the stator (defined in the 'Observer Benchmark') by its inverter. At the same time, the track of the reference speed trajectory (defined in the 'Observer Benchmark') is imposed to the IM by the connected also synchronous motor via its inverter. 
The parameters ${ }^{2} \lambda_{1}$ and $\lambda_{2}$, of observer (5) are chosen as $\lambda_{1}=1000, \lambda_{2}=800$.

Remark 2: Due to experimental conditions (temperature, ...), the identified parameters are not exactly the real parameters of $I M$. The control experimental conditions are nearly different compared to the identification conditions and moreover the identification methodology has a certain uncertainty in its results. Thus this case is already a first robustness test.

Speed estimation (Fig. 3)

On Fig. 3 the speed responses for identified parameters (nominal case) and case with stator resistance variation are shown. For robustness test case $\left(+50 \%\right.$ of $\left.R_{s}\right)$, the speed is affected a little compared to nominal case when the observer is under observable conditions and near unobservable conditions.

Load torque and flux estimation (Fig. 4 and 6)

Fig. 4 and 6 display the current and flux responses for identified parameters (nominal case) and case with stator resistance variation. The $+50 \%$ variation on $R_{s}$ affects clearly the flux response (Fig. 6) under observable and unobservable conditions according to the nominal case.

On the other hand, the tracking in load torque (Fig. 4) is not affected with this positive resistance variation when the $I M$ is under observable conditions but it is sensible a little on the unobservable conditions.
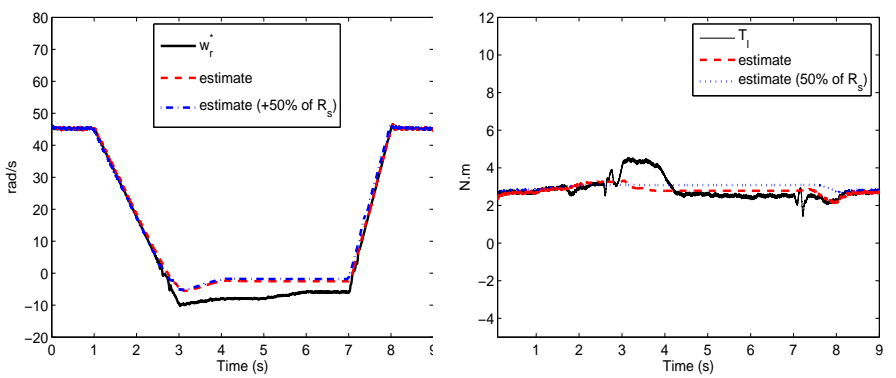

Fig. 3. $w_{r}$ and $\hat{w}_{r}$

Fig. 4. $T_{l}$ and $\hat{T}_{l}$
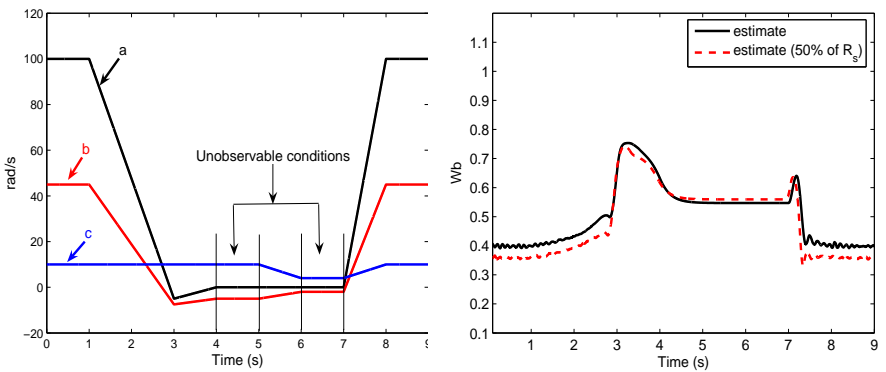

Fig. 5. Observer Benchmark Fig. 6. $\hat{\phi}_{r d}$

By comparing the experimental results obtained with the interconnected high gain observer given by [9], it can be remarked that the proposed sliding mode observer is stable near unobservable curve while the interconnected high gain becomes unstable. The main reason for this difference between the behavior of the two observers near unobservable curve lies in their estimation error gains. The proposed sliding mode observer uses gains which are preliminary fixed. The interconnected high gain observer uses gains which are computed

\footnotetext{
${ }^{2}$ guidelines parameters tuning are given in Appendix
}

at each iteration of the observer by solving Lyapunov equation.

\section{B. Part II: Observer-controller experimental results}

Now the proposed FOC-SMC using sliding mode observer in closed loop, is tested on an experimental set-up. A Sensorless Control Benchmark defines the adequate reference trajectories to evaluate the performances of the sensorless control algorithms under the following operating conditions (Fig. 7).

- Area 1. Low speed with nominal load (from 1s to $3 \mathrm{~s}$ ).

- Area 2. High speed with nominal load (from $4 s$ to $6 s$ ).

- Area 3. Very low speed (zero frequency) with nominal load (the IM is unobservable ${ }^{3}$ from $7 s$ to $9 s$ ).

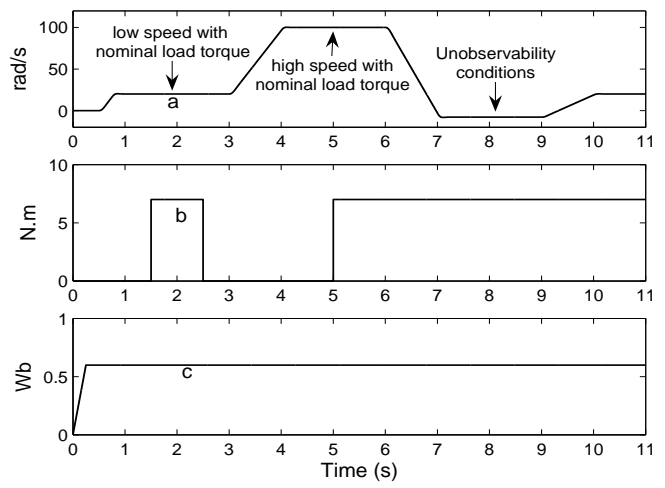

Fig. 7. Control benchmark

The parameters ${ }^{4}$ of controllers (10) and (25)-(27) are chosen as follows: $K p_{v d}=2, K p_{v q}=2, K_{I v d}=0.05, K_{\text {Ivq }}=0.05$, $\eta_{\phi_{r d}}=10, k_{\phi_{r d}}=-80, l_{\phi_{r d}}=4, \eta_{\omega_{r}}=5, k_{\omega_{r}}=-40$, $l_{\omega_{r}}=2$. Those of the observer are done in section (V-A).

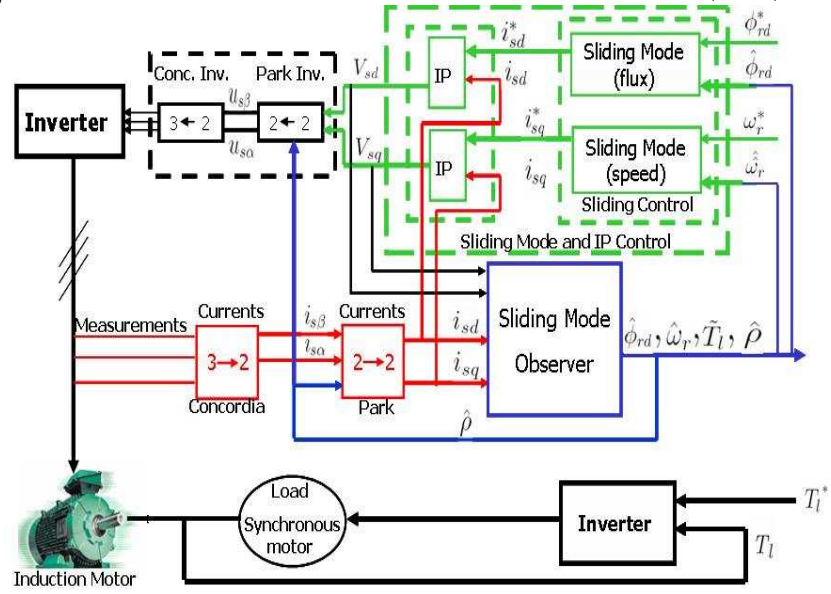

Fig. 8. Pie chart of the observer-controller

The block scheme chart used in experimental set-up to test the law control with observer in closed loop is presented in Fig. 8. The block "Sliding Mode Observer" uses only the stator current measurements in the reference rotating frame $(d-q)$ to estimate the currents, speed, flux amplitude and flux angle. The block "Sliding Mode and Field Oriented Control" contains the proposed controller. This block uses the estimates

\footnotetext{
${ }^{3}$ see section II-B for more details

${ }^{4}$ guidelines parameters tuning are given in Appendix
} 
of speed, flux amplitude and flux angle given by the block "Sliding Mode Observer" and the current measurements after using the transformation of Park and Concordia. Then, it gives the inputs control in the reference fixed frame $(a, b, c)$ after using the inverse transformations of Park and Concordia. These control inputs drive the inverter to impose the speed and flux reference trajectories (defined by the "Control Benchmark"). The track of the reference load torque trajectory (also defined in the "Control Benchmark") is imposed by the connected synchronous motor.

1) Case with identified parameters: Fig. 9 and 10 show the experimental results in case where identified parameters are used to design the flux and speed sliding mode control with sliding mode observer (observer-controller scheme).
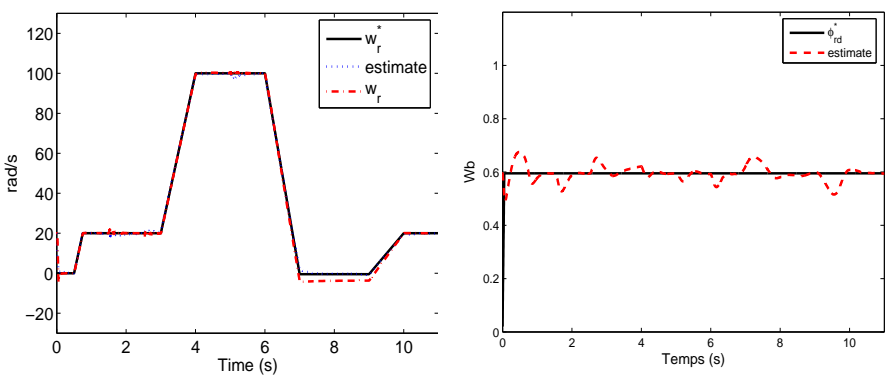

Fig. 9. $\omega_{r}^{*}, \omega_{r}, \hat{\omega}_{r}$.

Fig. 10. $\phi_{r d}^{*}, \hat{\phi}_{r d}$

We can remark that both systems "Control+Observer" give good performances i) in term of trajectory tracking: the motor speed (Fig. 9.b) tracks correctly its reference (Fig. 9.a) even under unobservable conditions (between 7 and $9 \mathrm{sec}$ ), nevertheless it appears a small static error when the motor is under unobservable conditions, ii) in term of perturbation rejection: the load torque is very well rejected under low speed and high speed (Fig. 9: 1.5 s, Fig. 9: $2.5 \mathrm{~s}$ and Fig. 9: 5 s). For estimated flux (Fig. 10), the same conclusion is given and moreover it exists a small peaking at the beginning (Fig. 10.b) which is due to initial conditions. Control efforts $V_{s d}$ and $V_{s q}$ are shown in figure (11). In figure 11 the measured and reference currents of $i_{s d}$ and $i_{s q}$ are displayed.
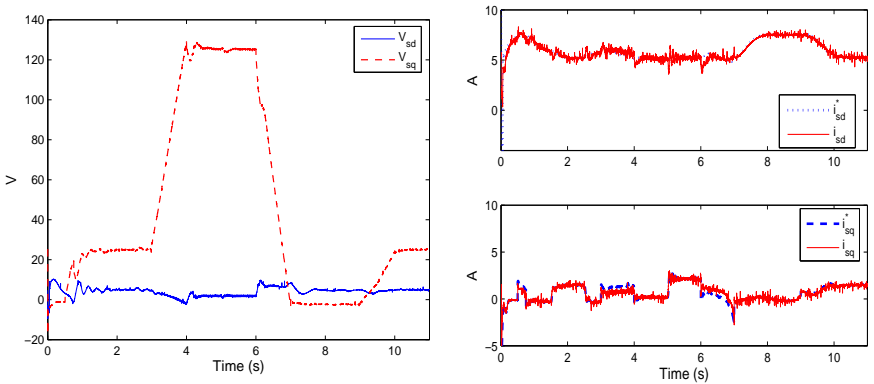

Fig. 11. $V_{s d}$ and $V_{s q}$

Fig. 12. $i_{s d}^{*}, i_{s d}$ and $i_{s q}^{*}, i_{s q}$

Remark 3: Remark (2) is introduced here.

2) Robustness case: To check the robustness of the designed Control-Observer with respect to motor parameters variation, a stator resistance variation of $+50 \%$ is considered. The results that we have obtained are depicted in Figures 13 and 14. It can be noticed that for motor speed (Fig. 13), the increase of the rotor resistance affects the performances of both "Control+Observer" at high and low speed conditions compared with the previous case. It appears a small static error at the time when the load torque is applied (Fig. 13: 1.5 $\mathrm{s}$ and Fig. 13: $5 \mathrm{~s}$ ) and when the load torque is removed (Fig. 13: $2.5 \mathrm{~s}$ ). When the motor is under unobservable conditions (between 7 and $9 \mathrm{sec}$ ), the static error is improved. For estimated flux (Fig. 14), the results are nearly similar compared with the previous case.
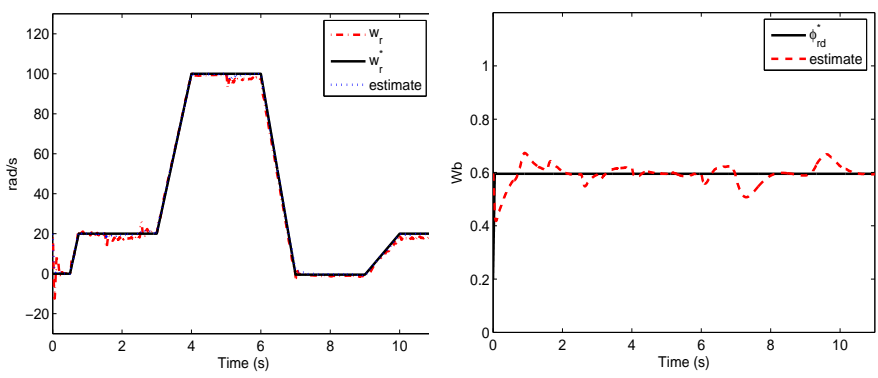

Fig. 13. $\omega_{r}^{*}, \omega_{r}, \hat{\omega}_{r}$.

Fig. 14. $\phi_{r d}^{*}, \hat{\phi}_{r d}$.

\section{CONCLUSION}

In this paper, a step by step sliding mode observer has firstly been designed to estimate the speed, flux and the load torque of $S I M$ even at very low speed conditions. Based on Lyapunov theory, the convergence of the proposed observer is proved. Experimental results show that the proposed observer is stable near and under unobservable conditions. The robustness of the proposed observer is verified according to $+50 \%$ stator resistance variation where it was found that the flux and speed estimations are sensible to this variation.

Secondly, a field oriented control combined with sliding mode controller is designed to steer the estimated speed and flux magnitude to their desired trajectories and stability analysis based on Lyapunov theory is given. Furthermore, sufficient conditions to guarantee the convergence of the whole control-observer system are presented.

The proposed controller using the designed observer in closed loop has been tested and validated by an experimental set-up using the reference trajectories of a realistic sensorless control benchmark. The robustness of the proposed controller using a sliding mode observer was experimentally verified with respect to significant test (resistance variation).

\section{ACKNOWLEDGMENTS}

Authors would like to thanks A. Glumineau and R. Boisliveau for the use of the experimental set-up located at IRCCyN (see [24]).

\section{REFERENCES}

[1] O. Barambones and A.J. Garrido "A sensorless variable structure control of induction motor drives", Electric Power Systems Research, 72, pp. 21-32, 2004.

[2] J-P. Barbot, M. Djemai, T. Boukhobza "Sliding Mode Observers" chapter 4, book "Sliding mode control in engineering", 2002.

[3] F. Blaschke, "The principle of field orientation applied to the new transvector closed-loop control system for rotating field machines", Siemens-Rev., 39, pp. 217-220, 1972.

[4] A. Boglietti, A. Cavagnino, L. Feraris, M. Lazzari, Energy-efficient motors, IEEE Industrial Electronics Magazine, vol.2, no.4, pp.32-37, 2008. 
[5] J. Chiasson, "Modeling and High-Performance Control of Electric Machines", IEEE Series on Power Engineering, Wiley-Interscience, 2005 .

[6] M. Comanescu, L. Xu, T. D. Batzel, "Decoupled Current Control of Sensorless Induction-Motor Drives by Integral Sliding Mode", IEEE Trans. on Industrial Electronics, vol. 55, no. 11, pp. 3836-3845, Nov 2008.

[7] G. Edelbaher, K. Jezernik, E., Urlep, 'Low-Speed Sensorless Control of Induction Machine", IEEE Transactions on Industrial Electronics, 35, 1, pp. 120-129, 2006.

[8] M. Feemster, P. Aquino, D. M. Dawson and A. Behal, "Sensorless rotor velocity tracking control for induction motors", IEEE Transactions on Control Systems Technology, 9, 4, pp. 645-653, 2001

[9] M. Ghanes, J. DeLeon, and A. Glumineau, "Observability Study and Observer-Based Interconnected Form for Sensorless Induction Motor", IEEE CDC, San Diego, California, USA, 2006.

[10] M. Ghanes, J. De Leon and A. Glumineau, "Cascade and highgain observers comparison for sensorless closed-loop induction motor control" IET CTA, vol. 2, no. 2, Page(s):133 - 150, Feb. 2008.

[11] L. Harnefors, M. Hinkkanen, "Complete Stability of Reduced-Order and Full-Order Observers for Sensorless IM Drives," IEEE Trans. on Industrial Electronics, vol. 55, no. 3, pp. 1319-1329, March 2008.

[12] J. Holtz, "Sensorless control of induction machines-with or without signal injection" IEEE Transactions on Industrial Electronics, 2006, 53, 1, pp. 7-30.

[13] V-M. Leppanen and J. Luomi, "Speed-Sensorless Induction Machine Control for Zero Speed and Frequency", IEEE Transactions on Industrial Electronics, 51, 5, 2004

[14] A. Isidori, "Nonlinear control systems", 3rd edition, Springer, Berlin, 1995.

[15] H. K. Khalil and G. Strangas, "Sensorless Speed Control of Induction Motor", American Control Conference, Boston, Massachusetts, USA, juin 30 - July 2, 2004.

[16] H. Kubota and K. Matsuse, "Speed sensorless filed-oriented control of induction motor with rotor resistance adaptation" IEEE Transactions on Industry Applications, 30, 5, pp.344-348, Mar./Apr., 1994.

[17] R. Marino, P. Tomei and C. M. Verrelli, "A nonlinear tracking control for sensorless induction motors "Automatica, 41, 6, pp. 1071-1077, 2005.

[18] M. Montanari, S. Peresada, A. Tilli, and A. Tonielli, "Speed sensorless control of induction motor based on indirect Field-orientation", Conference record of the 2000 IEEE industry applications conference, 3, pp. $1858-1865,2000$

[19] R. Pea, R. Cerdenas, J. Proboste, G. Asher, J. Clare, "Sensorless Control of Doubly-Fed Induction Generators Using a Rotor-CurrentBased MRAS Observer,' IEEE Trans. on Industrial Electronics, vol. 55, no. 1, pp. 330-339, Jan 2008

[20] A. Sabanovic and D.B. Izosimov, "Application of sliding modes to induction motor control", IEEE Transactions on Industry Applications, IA-17, pp.344-348, 1981.

[21] C.S. Staines, C. Caruana, M. Asher, "Sensorless Control of Induction Machines at Zero and Low Frequency Using Zero Sequence Currents", IEEE Transactions on Industrial Electronics, 53, 1, pp. 195-206, 2006.

[22] T. Tsuji, T. Hashimoto, H. Kobayashi, M. Mizuochi, K. Ohnishi, "A Wide-Range Velocity Measurement Method for Motion Control," IEEE Trans. on Industrial Electronics, vol. 56, no. 2, pp. 510-519, Feb 2009.

[23] V.I. Utkin, Sliding mode control design principles and applications to electric drives, IEEE Transactions on Industrial Electronics, 40, pp 2636, 1993.

[24] A. Glumineau, R. Boisliveau, (IRCCyN) and L. Loron, (IREENA) www.irccyn.ec-nantes.fr/hebergement/BancEssai/

[25] M. S. Zaky, M. M. Khater, S. S. Shokralla, H. A. Yasin, "WideSpeed-Range Estimation With Online Parameter Identification Schemes of Sensorless Induction Motor Drives", IEEE Trans. on Industrial Electronics, vol. 56, no. 5, pp. 1699-1707, May 2009.

\section{Appendix: Parameters tuning of the observer-controller scheme}

- The gains $\lambda_{1}$ and $\lambda_{2}$ of the observer (5) are chosen to satisfy equations (8) and (9) respectively.

- For the controller given by (10), the parameters $K p_{v d}, K p_{v q}, K_{I v d}$, $K_{\text {Ivq }}$ are determined as follows :

Considering the dynamic equations of $i_{s d}$ and $i_{s q}$ given by (1) without nonlinearities and coupling terms

$$
\left\{\begin{array}{l}
\dot{i}_{s d}=-\gamma i_{s d}+m_{1} u_{s d} \\
\dot{i}_{s q}=-\gamma i_{s q}+m_{1} u_{s q}
\end{array}\right.
$$

Writing the transfer function which lies the stator currents of (28) with their references given by (10) as a second order system in closed loop, it follows

$$
\left\{\begin{aligned}
\frac{i_{s d}}{i_{s d}^{*}} & =\frac{w_{n d}^{2}}{s^{2}+2 \zeta w_{n d}+w_{n d}^{2}} \\
\frac{i_{s q}}{i_{s q}^{*}} & =\frac{w_{n q}^{2}}{s^{2}+2 \zeta w_{n q}+w_{n q}^{2}}
\end{aligned}\right.
$$

By imposing $\zeta=1$ to avoid peaking and a currents bands-widths $F_{B D}$ at least less than a middle of $F_{e}=1 / T_{e}$ where $T_{e}=200 \mu \mathrm{s}$ is the sampling time:

$$
\left\{\begin{aligned}
\zeta & =1 \\
w_{n d} & =2 \pi F_{B D} \\
w_{n q} & =2 \pi F_{B D}
\end{aligned}\right.
$$

the parameters $K p_{v d}, K p_{v q}, K_{I v d}, K_{I v q}$ can be established:

$$
\left\{\begin{array}{rlrl}
K p_{v d}=\frac{2 \zeta-\gamma}{m_{1}}, & & T i_{v d}=\frac{2 \zeta-\gamma}{w_{n d}^{2}} \\
K p_{v q}=\frac{2 \zeta-\gamma}{m_{1}}, & T i_{v q}=\frac{2 \zeta-\gamma}{w_{n q}^{2}}
\end{array}\right.
$$

where $K_{I v d}=\frac{K p_{v d}}{T i_{v d}}$ and $K_{I v q}=\frac{K p_{v q}}{T i_{v q}}$

- For the controller given by (25)-(27), $l_{\phi_{r d}}>0, l_{\omega_{r}}>0$ and the parameters $k_{\phi_{r d}}, k_{\omega_{r}}, \eta_{\phi_{r d}}$ and $\eta_{\omega_{r}}$ are chosen to satisfy respectively (18), (24), (17) and (23).

Malek GHANES received the Dip.Ing. from T.O. M. Mammeri University in 2000,

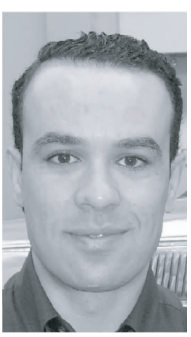
and received his M.S. degree and Ph.D. in applied automatic and informatics both from IRCCyN, Ecole Centrale Nantes, in 2002 and 2005, respectively. From sept. 2005 to sept. 2006, he was a Postdoctoral position at GReyC. Since sept. 2006, he is Associate Professor at ENSEA, France.

His research interests include observation and control of nonlinear system, with applications to electric and chaotic systems.

Gang ZHENG received his B.E. and M.E. degree both from Wuhan University in 2001 and 2004, respectively, and received his Ph.D. degree in Automatic control from ENSEA, France in 2006. From 2007, he held a postdoctroral position at INRIA, at LJK and at ENSEA, France.

His research interests include control and observation of nonlinear system, normal form and synchronization of chaotic systems. 